\title{
Die Kurfürstin im Alten Reich Korrespondenz und Klientel im 16. und 17. Jahrhundert
}

\author{
von \\ KATRIN KELLER
}

Die Sichtweise auf das Alte Reich als einen Kommunikationsraum, als ein interaktives
System ${ }^{1}$ hat in den letzten Jahren in der Forschung eine erhebliche Rolle gespielt,
wobei den Ausgangspunkt für diese Einschätzung vorrangig Untersuchungen zur
zweiten Hälfte des 16. Jahrhunderts bildeten. Deutlich herausgearbeitet worden ist
dabei vor allem, welche Rolle Kommunikationsprozesse auf verschiedenen Ebenen
beim Aushandeln von Kompromissen, für Wirkungsmöglichkeiten von Institutionen
des Reiches und damit für dessen Überlebensfähigkeit spielten, ${ }^{2}$ wie soziale Kom-
munikation dabei als konstitutives Element von Macht- und Herrschaftsverhältnissen
in Erscheinung trat. Neue Ansätze der Politikgeschichte zeigen hier einmal mehr die
Differenzen zwischen Verfassungsnormen und politischen Realitäten, führen prak-
tische und individuelle Handlungsbedingungen in konkreten Kontexten in neuer
Form vor Augen. ${ }^{3}$ Als Kommunikationsebenen, die in diesem Kontext eine Rolle spie-

1 Vgl. Georg Schmidt, Geschichte des Alten Reiches. Staat und Nation in der Frühen Neuzeit, München 1999, S. 11 f., 120 f., 142; Helmut NeuHaus, Das Reich in der Frühen Neuzeit (Enzyklopädie deutsche Geschichte 42), München 1997, S. 3-5.; THOMAS FRÖSCHL, „in frieden, ainigkaitt und ruhe beieinander sitzen“. Integration und Polarisierung in den ersten Jahren der Regierungszeit Kaiser Rudolfs II. 1576-1582, ungedruckte Habilitationsschrift Wien 1997, S. 56-62. Als Überblick zur neueren Literatur siehe: MATTHIAS SCHNETTGER, Von der „Kleinstaaterei“ zum „komplementären Reichs-Staat“. Die Reichsverfassungsgeschichtsschreibung seit dem Zweiten Weltkrieg, in: Hans-Christof Kraus/Thomas Nicklas (Hg.), Geschichte der Politik. Alte und neue Wege (Historische Zeitschrift, Beiheft 44), München 2007, S. 129-154; MAXIMILIAN LANZINNER, Einleitung, in: Ders./Arno Strohmeyer (Hg.), Der Reichstag 1486-1613: Kommunikation - Wahrnehmung - Öffentlichkeiten (Schriftenreihe der Historischen Kommission bei der Bayerischen Akademie der Wissenschaften 73), Göttingen 2006, S. 9-25; GABRIELE HAUG-MORITZ, Das Reich als medialer Kommunikationsraum. Skizze eines Forschungsprojektes, in: Frühneuzeit-Info 17 (2006), S. 58-69. Die in den letzten Jahren sehr zahlreichen Beiträge zu Begriff und Erscheinungsformen von „Kommunikation“ aus historischer Perspektive können hier nicht im Einzelnen nachgewiesen werden.

2 Vgl. z. B. MaXimilian LANZINNER, Friedenssicherung und politische Einheit des Reiches unter Kaiser Maximilian II. (1564-1576) (Schriftenreihe der Historischen Kommission bei der Bayerischen Akademie der Wissenschaften 45), Göttingen 1993; AlBRECHT P. LUTTENBERGER, Kurfürsten, Kaiser und Reich: politische Führung und Friedenssicherung unter Ferdinand I. und Maximilian II. (Veröffentlichungen des Instituts für europäische Geschichte Mainz, Abteilung Universalgeschichte 149; Beiträge zur Sozial- und Verfassungsgeschichte des Alten Reiches 12), Mainz 1994; SCHMIDT, Geschichte (wie Anm. 1), S. 113-115, 135; für Kursachsen siehe auch JOCHEN VÖTSCH, Kursachsen, das Reich und der mitteldeutsche Raum zu Beginn des 18. Jahrhunderts, Frankfurt am Main u. a. 2003.

$3 \mathrm{Vgl}$. für das Reich insbesondere Barbara StOllberg-Rilinger, Des Kaisers alte Kleider. Verfassungsgeschichte und Symbolsprache des Alten Reiches, München 2008; DiEs., Zeremoniell als politisches Verfahren. Rangordnung und Rangstreit als Struktur- 
len, sind dabei neben den Reichsinstitutionen selbst, innerhalb derer Kompromisse ausgehandelt wurden, sowie dem sich allmählich ausbildenden System von Gesandten beziehungsweise entsandten Räten vor allem die direkten Kommunikationsbeziehungen zwischen Fürsten zu erwähnen. Nicht zu vergessen sind in diesem Zusammenhang gedruckte Medien und geschriebene Zeitungen ${ }^{4}$ und natürlich die teilweise recht zahlreichen persönlichen Begegnungen - die persönliche Bekanntschaft zwischen Kaiser und Fürst war bis ins 17. Jahrhundert die Regel.

Betrachtet man diese Konstellation für die Beziehungen zwischen Dresden und Wien so erweist sich die Zeit zwischen Augsburgischem Religionsfrieden und Dreißigjährigem Krieg recht deutlich als Kulminationspunkt kommunikativer Beziehungen zwischen Reichsfürst und Kaiser, als eine Hochzeit von Kontakten auf ,persönlicher Ebene zwischen informeller Nachricht und offizieller Audienz. Dabei hatten die Mark Meißen und die Wettiner schon seit dem 14. Jahrhundert eine deutliche Annäherung an den Kaiser als Institution wie an die Familien der Luxemburger beziehungsweise Habsburger durchlaufen. Der Erwerb des Kurfürstentums Sachsen 1423 verlieh dem in besonderer Weise Ausdruck. Das Reformationszeitalter stellte in verschiedener Hinsicht einen Bruch in dieser Entwicklung dar, aber nicht zuletzt aufgrund der Jugendbekanntschaft zwischen Maximilian II. und Kurfürst August sowie deren individueller religiöser Prägung blieben Rahmenbedingungen für eine intensive Kommunikation zwischen beiden Fürsten gegeben. ${ }^{5}$ Der Facettenreichtum der jahrzehntelangen Korrespondenz beider Fürsten verdiente eine intensivere Untersuchung. ${ }^{6}$

merkmale des frühneuzeitlichen Reichstags, in: Zeitschrift für Historische Forschung 19 (1997), S. 91-132; Ronald Asch/Dagmar FreIsT (Hg.), Staatsbildung als kultureller Prozess. Strukturwandel und Legitimation von Herrschaft in der Frühen Neuzeit, Köln/ Weimar/Wien 2005; RUdOlF SCHLÖGL, Politik- und Verfassungsgeschichte, in: Joachim Eibach/Günther Lottes (Hg.), Kompass der Geschichtswissenschaft, Göttingen 2002, S. 95-111; Barbara StOllberg-Rilinger, Einleitung, in: Dies. (Hg.), Was heißt Kulturgeschichte des Politischen? (Zeitschrift für Historische Forschung, Beiheft 35), Berlin 2005, S. 9-24; Hillard von Thiessen/Christian Windler (Hg.), Akteure der Außenbeziehungen. Netzwerke und Interkulturalität im historischen Wandel, Köln/Weimar/Wien 2010.

4 Vgl. Andreas Würgler, Medien in der Frühen Neuzeit (Enzyklopädie deutsche Geschichte 85), München 2009; CORNEL ZwIERLEIN, Fuggerzeitungen als Ergebnis von italienisch-deutschem Kulturtransfer 1552-1570, in: Quellen und Forschungen aus italienischen Archiven und Bibliotheken 90 (2010), S. 169-224; OSWALD BAUER, Zeitungen vor der Zeitung. Die Fuggerzeitungen (1568-1605) und das frühmoderne Nachrichtensystem (Colloquia Augustana 28), Berlin 2011, S. 31-50; JohannEs ARnDT/EstHer-BEATE KöRBER (Hg.), Das Mediensystem im Alten Reich der Frühen Neuzeit (1600-1750) (Veröffentlichungen des Instituts für Europäische Geschichte Mainz, Beiheft 75), Göttingen 2010.

5 Vgl. Robert Holtzmann, Kaiser Maximilian II. bis zu seiner Thronbesteigung (1527-1564). Ein Beitrag zur Geschichte des Übergangs von der Reformation zur Gegenreformation, Berlin 1903, S. 33, 240, 425 f.; LANZINNER, Friedenssicherung (wie Anm. 2), S. 73, 309, 358-360, 412; PAula SutTer-FiChTNER, Emperor Maximilian II., New Haven/ London 2001, S. 14, 128; Friedrich Edelmayer, Söldner und Pensionäre. Das Netzwerk Philipps II. im Heiligen Römischen Reich (Studien zur Geschichte und Kultur der iberischen und iberoamerikanischen Länder 7), München 2002, S. 216; Jens BrUNING, August. 1553-1586, in: Frank-Lothar Kroll (Hg.), Die Herrscher Sachsens. Markgrafen, Kurfürsten, Könige. 1089-1918, München 2007, S. 110-125.

6 Sächsisches Staatsarchiv - Hauptstaatsarchiv Dresden (im Folgenden: HStA Dresden), 10024 Geheimes Archiv, Loc. 8501/1; Loc. 8500/1; Loc. 8499/7-10; Loc. 10297/14; Loc. $8500 / 3$ und weitere. Einen (unvollständigen) Überblick über Korrespondenzpartner und 
Ergänzt wurde der schriftliche Austausch der beiden Fürsten im Übrigen durch eine auffällig dichte Folge direkter persönlicher Treffen, die nicht nur im Umfeld von Kaiser- beziehungsweise Königswahl und Reichstagen stattfanden. Maximilian II. kam auch zweimal nach Dresden, während der Kurfürst nach 1553 mindestens dreimal in Prag und einmal in Wien weilte. ${ }^{7}$

Nach 1576 und vor allem während der Regierungszeit von Kurfürst Christian I. von Sachsen mit seiner politischen Annäherung an Frankreich ${ }^{8}$ ließ die Intensität direkter fürstlicher Korrespondenz nach. Sein älterer Sohn Christian II. dagegen nahm zu Beginn des 17. Jahrhunderts wieder engeren Kontakt zum Kaiser auf, den auch zwei Besuche des jungen Kurfürsten bei Rudolf II. dokumentieren. Beide Fürsten verband nicht zuletzt ihre Sammelleidenschaft und Kunstbegeisterung. ${ }^{9}$ Der bekannte Besuch Kaiser Matthias' und König Ferdinands II. 1617 in Dresden bei Kurfürst Johann Georg I. zeigt die anhaltende Relevanz persönlicher Kontakte, die sich auch in der Folgezeit im Rahmen der Beziehungen zwischen Habsburgern und Wettinern abzeichnet: ${ }^{10}$ Angesichts der sinkenden Bedeutung von Reichstagen und Wahlen als institutionalisierten Treffpunkten waren fürstliche Treffen zwar in ihrer Zahl im 17. Jahrhundert deutlich rückläufig, durch Besuche der sächsischen Kurfürsten in Prag beziehungsweise Leitmeritz $(1638,1652)$, später durch Inkognito-Reisen kurfürstlicher Prinzen nach Wien $(1678,1688,1691)$ blieb eine persönliche Ebene des Kontakts zwischen den Kurfürsten von Sachsen und dem Kaiserhaus jedoch dauerhaft erhalten, bis 1719 wieder familiäre Verbindungen hergestellt wurden.

Innerhalb des Kommunikationsraumes Altes Reich ${ }^{11}$ spielten jedoch keineswegs nur Männer eine Rolle, auch wenn sie natürlich die Mehrheit derer darstellten, durch

-partnerinnen Kurfürst Augusts gibt das Findbuch zum Teilbestand Handschreiben im Geheimen Archiv (fol. 11-25c) im Hauptstaatsarchiv Dresden; vgl. auch WiELAND HeLD, Die politische Korrespondenz des sächsischen Kurfürsten August (1553-1586). Ein Editionsdesiderat, in: NASG 70 (1999), S. 237-244.

7 Vgl. KATRIN KELLER, „Alhier an dem kayserlichen hofe ist wenig newes vorgefallen, welches zu berichten der importanz were ...“. Verbindungen zwischen den Residenzen Wien und Dresden im 17. Jahrhundert, in: Werner Paravicini/Jörg Wettlaufer (Hg.), Vorbild, Austausch, Konkurrenz. Höfe und Residenzen in der gegenseitigen Wahrnehmung (Residenzenforschung 23), Ostfildern 2010, S. 137-155, hier S. 145 f., 155; HaRRIET Rudolph, Das Reich als Ereignis. Formen und Funktionen der Herrschaftsinszenierung bei Kaisereinzügen (1558-1618) (Norm und Struktur 38), Köln/Weimar/Wien 2011, S. 165 f.

8 Vgl. Thomas Klein, Der Kampf um die zweite Reformation in Kursachsen 15861591 (Mitteldeutsche Forschungen 25), Köln/Graz 1962, S. 129-136.

9 Vgl. Thomas Da Costa Kaufmann, Höfe, Klöster und Städte. Kunst und Kultur in Mitteleuropa 1450-1800, Köln 1998, S. 209 f., 233; Damian DombrowsKi, Dresden - Prag. Italienische Achsen in der zwischenhöfischen Kommunikation, in: Barbara Marx (Hg.), Elbflorenz. Italienische Präsenz in Dresden 16.-19. Jahrhundert, Amsterdam/Dresden 2000, S. 65-100; Helen Watanabe-O'Kelly, Court Culture in Dresden. From Renaissance to Baroque, Basingstoke 2002, S. 90 f., 104-106; DiRK SYNDRAM, Von fürstlicher Lustbarkeit und höfischer Repräsentation. Die Kunstkammer und die Dresdner Sammlungen der Renaissance, in: Ders./Antje Scherner (Hg.), In fürstlichem Glanz. Der Dresdner Hof um 1600, Dresden 2004, S. 54-69, hier S. 65 f.

10 Vgl. HStA Dresden, 10024 Geheimes Archiv, Loc. 10736/33, Der Kaiser Zusammenkunft mit den Kurfürsten zu Sachsen, bes. Besuche letzterer in kaiserlichen Hoflagern Treffen, undat. [1348-1723].

11 Vgl. KatRin KelleR, Kommunikationsraum Altes Reich. Zur Funktionalität der Korrespondenznetze von Fürstinnen im 16. Jahrhundert, in: Zeitschrift für Historische Forschung 31 (2004), S. 205-230. 
deren Reisen, Schriften, Briefe, Gespräche dieser Raum konstituiert und perpetuiert wurde. Auf die Ergänzung und Erweiterung ,männlicher' fürstlicher Kommunikation durch ein ,weibliches' Gegenstück, durch Kontakte zwischen Fürstinnen, ${ }^{12}$ möchte ich hier anhand von Beispielen aus Dresden ausführlicher eingehen. Im Zentrum stehen dabei zwei Kurfürstinnen: zum einen Kurfürstin Anna von Sachsen (1532-1585), eine geborene Prinzessin von Dänemark, erste Gemahlin Kurfürst Augusts. ${ }^{13}$ Deren außerordentlich umfangreiche Korrespondenz, für die Kopiale auch die ausgehenden Schreiben dokumentieren, ist in Dresden fast vollständig überliefert und zuletzt auch teilweise ausgewertet worden. Bislang kaum genutzt wurde dagegen die Korrespondenz von Kurfürstin Magdalena Sibylla (1612-1687), geborene Markgräfin von Brandenburg-Bayreuth, Gemahlin Kurfürst Johann Georgs II. ${ }^{14}$ Sie umfasst allerdings mit etwa 2.500 Schreiben an die Kurfürstin nur einen Bruchteil des für Kurfürstin Anna überlieferten Materials, ${ }^{15}$ die ausgehende Korrespondenz der Fürstin ist in Dresden nicht überliefert. Vom Überlieferungsumfang her wäre auch ein Vergleich der Korrespondenz von Kurfürstin Anna mit der ihrer Schwiegertochter Sophie (1568-1622), einer geborenen Prinzessin von Brandenburg, möglich gewesen. Bei den für sie erhaltenen Briefkonvoluten ${ }^{16}$ handelt es sich aber fast ausschließlich um Korrespondenzen aus ihrer Witwenzeit; Konzepte ausgehender Briefe sind auch in diesem Fall nur sporadisch in den Akten enthalten. Außerdem unterlag zu Lebzeiten von Kurfürstin Sophie die Verbindung zum Kaiserhof speziellen Bedingungen, weil es über Jahrzehnte keine Kaiserin gab, die als Korrespondenzpartnerin zur Verfügung gestanden hätte. ${ }^{17}$

12 Zum Verhältnis von Politikgeschichte und Geschlechtergeschichte für die Frühe Neuzeit siehe die Beiträge in: Hillard vON Thiessen/Christian Windler (Hg.), Akteure der Außenbeziehungen. Netzwerke und Interkulturalität im historischen Wandel (Externa 1), Köln/Weimar/Wien 2010.

13 Zur Person der Fürstin siehe Karl von Weber, Anna, Churfürstin von Sachsen, Leipzig 1865; KONRAD STURMHÖFEL, Kurfürstin Anna von Sachsen. Ein politisches und sittengeschichtliches Lebensbild aus dem 16. Jahrhundert, Leipzig 1905; PERNILLE ARENFELDT, The Political Role of the Female Consort in Protestant Germany, 1550-1585. Anna of Saxony as „Mater Patriae“, Diss. Masch. Florenz 2005; KATRIn Keller, Kurfürstin Anna von Sachsen (1532-1585), Regensburg 2010.

14 Die Handschreiben an Kurfürstin Magdalena Sibylla von Sachsen sind überliefert in: HStA Dresden, 10024 Geheimes Archiv, Loc. 8567/4-7; Loc. 8568/1-5; Loc. 8569/1-4; Loc. 8570/1-4. Zu ihrer Person siehe Franz OTTO STICHART, Galerie der sächsischen Fürstinnen, Leipzig 1857, S. 376-388; einige Hinweise auch bei UTE ESSEGERN, Fürstinnen am kursächsischen Hof. Lebenskonzepte und Lebensläufe zwischen Familie, Hof und Politik in der ersten Hälfte des 17. Jahrhunderts: Hedwig von Dänemark, Sibylla Elisabeth von Württemberg und Magdalena Sibylla von Preußen (Schriften zur sächsischen Geschichte und Volkskunde 19), Leipzig 2007, S. 380 f., 400.

15 Vgl. Arenfeldt, Political Role (wie Anm. 13), S. 38, 43-45.

16 Die Handschreiben an Kurfürstin-Witwe Sophia von Sachsen siehe in: HStA Dresden, 10024 Geheimes Archiv, Loc. 8543/6-9; Loc. 8544/1-8; Loc. 8545/1-12; Loc. 8546/1-8; Loc. 8547/1-4. Zu ihrer Person siehe STICHART, Galerie (wie Anm. 14), S. 299-316; erwähnt wird sie auch in KleIN, Zweite Reformation (wie Anm. 8), passim; zu ihrer Zeit als Witwe Hinweise bei Essegern, Fürstinnen am kursächsischen Hof (wie Anm. 14), S. 47 f., 60-63, 74 f., 102-105, 244 f., 266, 278; KaTRIN Keller, Familie und Politik: Die sächsischen Kurfürstinnen in der zweiten Hälfte des 16. Jahrhunderts, in: Helmar Junghans (Hg.), Die sächsischen Kurfürsten als Erben des Kurfürsten Moritz von Sachsen während des Religionsfriedens von 1555 bis 1618 (Quellen und Forschungen zur sächsischen Geschichte 31), Berlin 2007, S. 279-296, hier S. 292-295.

17 Auch der Briefwechsel von Herzogin Elisabeth von Sachsen, der derzeit in einer Edition zugänglich gemacht wird, scheint keine Verbindungen zur Gemahlin König Ferdi- 
Diese Rahmenbedingungen ebenso wie der größere zeitliche Abstand zwischen Anna und Magdalena Sibylla und schließlich ihrer beider Position als Gemahlin eines regierenden Fürsten ließen deshalb den Vergleich dieser beiden Beispiele sinnvoller erscheinen. Für beide Fürstinnen wird zunächst kurz die Ausdehnung ihrer Korrespondenzbeziehungen innerhalb des Alten Reiches skizziert und dann speziell ihren Verbindungen nach Wien nachgegangen. Neben Einblicken in Entwicklungstendenzen sollte es der Vergleich insbesondere ermöglichen, beide Fürstinnen und ihre Korrespondenznetze hinsichtlich individueller Prägungen und Zeitumstände zu relativieren und damit einige allgemeinere Beobachtungen zu brieflichen Netzwerken von Fürstinnen im Alten Reich zu formulieren.

\section{$I$.}

Eine erste Übersicht über die Korrespondenzpartner und -partnerinnen, ${ }^{18}$ mit denen Kurfürstin Anna in der zweiten Hälfte des 16. Jahrhunderts anhaltende, umfangreiche Briefwechsel verbanden, zeigt zwei zentrale Charakteristika: Es handelte sich um ein überwiegend, wenn auch keineswegs ausschließlich weibliches Korrespondenznetz, und dieses Netz erfasste zumindest zum Zeitpunkt seiner größten Ausdehnung um 1580 das Alte Reich fast zur Gänze. Zu den regelmäßigen und damit wohl auch relativ bedeutsamen Adressaten beziehungsweise Absendern von Briefen gehörten zwischen 1551 und 1585 rund 100 Personen; davon waren gut zwei Drittel Frauen. Wenn man die Dauer der brieflichen Kontakte genauer betrachtet, wird die Dominanz der Verbindungen zu anderen Frauen fürstlicher und hochadliger Herkunft noch deutlicher. Während sich knapp die Hälfte aller Korrespondenzen mit Männern nur über weniger als drei Jahre erstreckte, hielten briefliche Kontakte mit Frauen fast in der Hälfte aller Fälle zwischen sieben und 15 Jahre. Noch einmal ein Viertel der Frauenbriefwechsel erstreckte sich zudem über mehr als zwanzig Jahre, während das bei den männlichen Briefschreibern nur in ganz wenigen Fällen zu beobachten ist. Diese langährigen Korrespondenten der Kurfürstin Anna gehörten dabei ihrer nächsten Verwandtschaft an; es handelte sich um ihre Brüder Friedrich von Dänemark (1534-1588) und Johann von Holstein-Sonderburg (1545-1622) sowie ihren Onkel Herzog Johann von Holstein (1521-1580). Nur Landgraf Wilhelm von Hessen-Kassel (1532-1592) bildete eine Ausnahme, aber über seine Mutter war auch er mit den Albertinern verwandt.

Bei den Korrespondentinnen waren es ebenfalls vier Personen, mit denen die Kurfürstin am längsten in Verbindung stand: Mit den Gräfinnen Anna von Hohenlohe $(† 1594)$ und Agnes von Solms († 1588), mit Burggräfin Katharina Dorothea von Meißen (1538-1604), Annas Nichte, und Herzogin Elisabeth von Mecklenburg-Güstrow (1524-1586), ihrer Tante, verbanden die Kurfürstin über dreißig Jahre währende Briefwechsel. Unter den anderen elf Frauen, mit denen die Kurfürstin über mehr als zwanzig Jahre korrespondierte, befanden sich mit Markgräfin Emilia von Brandenburg-Ansbach (1516-1591) und Herzogin Sidonia von Braunschweig-Calenberg (1518-1575) zwei ihrer Schwägerinnen, außerdem ihre jüngere Schwester (Herzogin Dorothea von Braunschweig-Lüneburg, 1543-1617) sowie zwei Nichten. Aber mit Herzogin Anna von Bayern (1528-1590), Kurfürstin Sabina von Brandenburg (1529-

nands I. zu enthalten: André ThIEme (Hg.), Die Korrespondenz der Herzogin Elisabeth von Sachsen, Erster Band: Die Jahre 1505 bis 1532 (Quellen und Materialien zur sächsischen Geschichte und Volkskunde 3/1), Leipzig 2010.

18 Vgl. Keller, Kommunikationsraum (wie Anm. 11), S. 216-219, 229 f.; ArenfeldT, Political Role (wie Anm. 13), S. 43-57. 
1574), Gräfin Dorothea von Mansfeld (1493-1578) und der dänischen Hofdame Anna von Hardenberg $(† 1589)$ findet man hier auch Frauen, mit denen Kurfürstin Anna offenbar jenseits von familiären Beziehungen jahrzehntelange Briefwechsel verbanden.

Angesichts der Dauerhaftigkeit vor allem der Frauenbriefwechsel kann es nicht verwundern, dass sich die größte Zahl von Briefpartnern und -partnerinnen der Kurfürstin für die Zeit um 1580 ausmachen lässt. Trotz der weiten Streuung ihrer Kontakte wird erkennbar, dass die Schwerpunkte der Korrespondenzbeziehungen von Kurfürstin Anna zu dieser Zeit im nördlichen, im protestantischen Reichsgebiet lagen. Mit verschiedenen Zweigen des Hauses Braunschweig und den brandenburgischen Kurfürsten und Markgrafen waren die Kontakte über Jahrzehnte recht eng. Sowohl im Fall von Braunschweig-Lüneburg, wohin Annas jüngere Schwester geheiratet hatte, wie mit dem kurfürstlichen Brandenburg, von wo 1582 ihre Schwiegertochter Sophie nach Dresden kam, spielten familiäre Beziehungen dabei eine Rolle. Familiäre Korrespondenz war es auch im Wesentlichen, die Anna mit Holstein, Mecklenburg und natürlich mit dem dänischen Königshaus verband. Auch für die Kurpfalz gilt das, denn die älteste Tochter der Kurfürstin, Elisabeth (1552-1590), ehelichte 1570 Pfalzgraf Johann Casimir (1543-1592).

Unter den dauerhaften Korrespondenzen mit nicht direkt verwandten fürstlichen Häusern waren die intensiven Kontakte zum benachbarten Anhalt besonders auffällig; außerdem die bereits erwähnte Korrespondenz mit Wilhelm von Hessen-Kassel und dessen Ehefrau Sabina von Württemberg (1549-1581) sowie zu verschiedenen Habsburgerinnen. Die ausgewiesenen Kontakte reichten bei den Korrespondentinnen der Kurfürstin also deutlich in das katholische Gebiet hinein und erschlossen damit das Reichsgebiet sehr weitgehend, wobei freilich eine inhaltliche Gewichtung erst aufgrund detaillierterer Auswertungen möglich wäre. Neben Kaiserin Maria (1528-1603) und Herzogin Anna von Bayern, der ältesten Tochter Kaiser Ferdinands I., war es nach 1581 vor allem deren Tochter Maria (1551-1608), seit 1571 mit Erzherzog Karl von Innerösterreich verehelicht, mit denen Anna regelmäßig Schreiben und Präsente wechselte.

\section{$I I$.}

Unter diesen Kontakten der Kurfürstin ins katholische Reichsgebiet spielte die Verbindung zu Kaiserin Maria de Austria (1528-1603), einer Tochter Karls V., natürlich eine besondere Rolle. ${ }^{19}$ Maria war seit 1548 mit Maximilian II. verheiratet; nach seinem Tod lebte sie noch einige Jahre in Prag und reiste dann 1581 zurück nach Spanien, wo sie sich meist in Madrid im Kloster Descalzas Reales aufhielt. ${ }^{20}$ Kurfürstin Anna und Königin Maria lernten sich vermutlich anlässlich der Wahl und Krönung Maximilians zum König 1562 in Frankfurt am Main kennen; zumindest sind in diesem Zusammen-

$19 \mathrm{Vgl}$. zu diesem Briefwechsel KATRIN KELLER, Le pouvoir des lettres: les réseaux de communication entre Dresde et Vienne au XVIe siècle, in: Isabelle Poutrin/Marie-Karine Schaub (Hg.), Femmes \& pouvoir politique. Les princesses d'Europe. XVe-XVIIIe siècle, Rosny-sous-Bois 2007, S. 164-181.

$20 \mathrm{Zu}$ ihrer Zeit in Madrid vgl. Magdalena S. SÁncheZ, The Empress, the Queen and the Nun. Women and Power at the Court of Philip III. of Spain, Baltimore 1998; SUTTERFichtNer, Maximilian II. (wie Anm. 5), S. 19 f., 116-118, 200, 203, 211-213; HelgA Widorn, Die spanischen Gemahlinnen der Kaiser Maximilian II., Ferdinand II. und Leopold I., Diss. Masch. Wien 1959, S. 1-51. 
hang Treffen zwischen den Fürstinnen wie zwischen den Ehepaaren belegbar. ${ }^{21}$ Eine Korrespondenz der beiden lässt sich jedoch erst ab 1570 nachweisen, nach einem Besuch des sächsischen Fürstenpaares in Prag. In der Zeit bis zum Tod der Kurfürstin Anna sind dann 46 Briefe zwischen beiden erhalten, wobei die Korrespondenz nach Marias Rückzug nach Spanien deutlich rückläufig war, ohne allerdings ganz abzubrechen. ${ }^{22}$

Die Briefe zeigen zunächst eine gewisse Unsicherheit der Kurfürstin, die wohl in erster Linie aus dem hierarchischen Verhältnis zwischen den Korrespondentinnen resultierte, das Anna durch die Ansprache des Kaiserpaares als obrigkeit ${ }^{23}$ auch direkt thematisierte. Inhaltlich ist die Korrespondenz vor allem dadurch geprägt, dass die Kurfürstin der Kaiserin immer wieder Gaben übermittelte, worauf diese im Gegenzug mit Dank und einer Versicherung ihrer freundlichen Neigung gegenüber der Absenderin reagierte. Bei diesen Geschenken handelte es sich in erster Linie um Heilmittel für Maria selbst oder den Kaiser wie das begehrte Aquavitae der Kurfürstin sowie um eingemachte Früchte; beides sind Gaben, die insgesamt im Umfeld der Briefwechsel der Kurfürstin eine erhebliche Rolle spielten. ${ }^{24}$ Dass aus diesen Gaben eine gewisse Verpflichtung der Kaiserin resultierte, formulierte diese selbst: Vnd wo wir berwiderumben in vnser gwalt ichtwas wißen, damitt derselben in gleichen vnnd mehrerm zur annemblicheitt gedient sein, oder wir sonsten sölche euer Liebden ertzaigung widergelten möchte, sollte es gwißlichen euer Liebden zu aller zeitt offen vnnd vnuersagt sein, vnnd wöllen euer Liebden dasselb von vnns zubegern keinen scheich haben [...]. ${ }^{25}$

Derartige Gegendienste leistete die Kaiserin zum einen ihrerseits durch Gaben, wie zum Beispiel Samen in Spanien heimischer Pflanzen oder - nach einem Besuch der kaiserlichen Familie in Dresden 1575 - mit einem Bett samt zugehöriger Tapisserien. ${ }^{26}$ Zum anderen konnte aber die Kurfürstin auf die Dankbarkeit der Kaiserin zurückgreifen, wenn sie selbst etwas am Kaiserhof beziehungsweise beim Kaiser erreichen wollte. So bat Anna etwa 1572 die Kaiserin um Unterstützung, als Herzogin Sidonia von Braunschweig-Calenberg (1518-1575), Annas Schwägerin, in einem Ehestreit ihr Recht beim Kaiser suchen wollte, durch demutige vorbitt ${ }^{27}$ Marias in den Angelegenheiten ihrer Schwägerin beim Kaiser, die auch gewährt wurde.

Aber auch die Kaiserin ihrerseits griff auf derartige Unterstützung durch die Kurfürstin zurück, so etwa indem sie 1582 aus Lissabon darum bat, dass die Kurfürstin doch ihren Ehemann darin bestärken möge, Marias Sohn, Kaiser Rudolf II., während des Reichstags und insgesamt weiterhin zu unterstützen. Anna antwortete darauf: [...] vnnd mögen euer Mayestet wol gewis darfür haltten, das seine Liebden [Kurfürst

21 Die Krönungen Maximilians II. zum König von Böhmen, Römischen König und König von Ungarn (1562/63) nach der Beschreibung des Hans Habersack, ediert nach CVP 7890 von Friedrich Edelmayer u. a. (Fontes rerum Austriacarum 1/13), Wien 1990, S. $146,155,162,177$.

22 Zum größeren Teil befindet sich diese Korrespondenz zusammengefasst in: HStA Dresden, 10024 Geheimes Archiv, Loc. 8538/9 (1575-1585).

23 So z. B. HStA Dresden, 10024 Geheimes Archiv, Copial 514, fol. 155v (14. Juli 1570).

24 Vgl. Keller, Kurfürstin Anna (wie Anm. 13), S. 100-102, 166 f.

25 HStA Dresden, 10024 Geheimes Archiv, Loc. 8538/9, fol. 39r (15. August 1570).

26 Vgl. HStA Dresden, 10024 Geheimes Archiv, Copial 516, fol. 40r/v (9. April 1570); Copial 519, fol. 39r-40v (10. Juni 1575).

27 HStA Dresden, 10024 Geheimes Archiv, Copial 516, fol. 58r/v (16. Mai 1572); Loc. 8538/9, fol. 42r-43r (23. Juni 1572). Zu Sidonia siehe ANDREA LILIENTHAL, Die Fürstin und die Macht. Welfische Herzoginnen im 16. Jahrhundert: Elisabeth, Sidonia, Sophia (Quellen und Darstellungen zur Geschichte Niedersachsens 127), Hannover 2007, S. 183-240. 
August] sich gegen irer Mayestet nichts weniger als gegen derselben geliebten herrn vattern, weilant euer Maiestet geliebten herrn und gemal hochlöblicher seeliger gedechtnus, aller vnterthenigsten dinstwilligkeit bevleissigen werden, wie sie dan solches auch diesen reichstag obn rubm im werck also bewiesen haben. Vnnd do ich kunffig vor mein person ichtwas erinnern kann, welches irer Mayestet zum besten gereichen mag, will ich an mir nichts erwindenn lassenn. ${ }^{28}$

Der Briefwechsel dokumentiert also in erster Linie die Herstellung und Perpetuierung gegenseitiger Verpflichtungen zwischen zwei Fürstinnen, die eindeutig auf Initiative der Kurfürstin zustande kamen: Anna nahm die Korrespondenz auf und leitete die damit verbundene Intensivierung der Beziehung zwischen den beiden Frauen durch Geschenke ein. Die Kaiserin reagierte darauf positiv, wobei vor allem durch die Weiterführung des Briefwechsels nach dem Tod des Kaisers und sogar nach ihrer Rückkehr nach Spanien die Bedeutung, die Maria diesem Kontakt beimaß, unterstrichen wird. Für beide Seiten resultierte aus der mit den Briefen dokumentierten Beziehung eine Möglichkeit, über Dritte in Angelegenheiten von politisch-familiärem Interesse Einfluss zu nehmen. Anhand dieses Beispiels wird die Einbeziehung von Fürstinnen in das oben angesprochene, das Alte Reich umspannende kommunikative Netzwerk erkennbar, in dem Frauen Kontakte zwischen Männern verstärken oder erweitern konnten. ${ }^{29}$

Dabei bleibt für unser Beispiel hervorzuheben, dass es sich hierbei nicht um eine wirklich vertraute Korrespondenz zwischen Fürstinnen handelte. Im Vergleich zu Annas Kontakten zu anderen Frauen, unter den Habsburgerinnen etwa zu Anna von Bayern, ${ }^{30}$ war der Briefwechsel mit der Kaiserin relativ kurz und sporadisch. Für die Bewertung und Einordnung dieses Briefwechsels ist es jedoch wesentlich, darauf hinzuweisen, dass es sich hier nur um einen von mehreren Korrespondenzsträngen handelt, den die sächsische Kurfürstin in das Umfeld der kaiserlichen Familie unterhielt. So wie die Korrespondenzen von Fürsten mehrere Ebenen aufwiesen - ,persönliche beziehungsweise eigenhändige Korrespondenz, Staatskorrespondenz, Kontakte über Dritte, vor allem fürstliche Räte und Gesandte -, so umfasste auch das Beziehungsnetz der Kurfürstin zum Kaiserhof mehrere Ebenen. Neben dem Briefwechsel mit der Kaiserin selbst und aufs Engste mit diesem verbunden gab es zwischen 1563 und 1585 immer auch mindestens einen weiteren engen Kontakt mit einer Dame der Wiener höfischen Gesellschaft, die das Vertrauen der Kaiserin genoss.

Den Anfang machte dabei Brigitta Trautson (1505/10-1576), die Frau des einflussreichen kaiserlichen Geheimen Rates und Obersthofmeisters Hans Trautson (um 1507-1589), mit der die Kurfürstin zwischen 1563 und 1576 in Verbindung stand. ${ }^{31}$ Dabei ist die Vermittlerrolle hervorzuheben, die Brigitta Trautson in Annas Beziehung zu Kaiserin Maria zukam: Während in den ersten Jahren dieser Aspekt verbal noch keine Rolle spielte, erscheint ab 1567 sehr häufig die Bitte der Kurfürstin, Grüße an die

28 HStA Dresden, 10024 Geheimes Archiv, Loc. 8534/1, fol. 254r (3. August 1582); Loc. 8538/9, fol. 22r (21. August 1582).

29 Vgl. Keller, Kurfürstin Anna (wie Anm. 13), S. 105-111; Keller, Kommunikationsraum (wie Anm. 11), S. $226 \mathrm{f}$.

30 Vgl. HStA Dresden, 10024 Geheimes Archiv, Loc. 8534/1 (1571-1584); Loc. 8536/1 (1575-1585).

$31 \mathrm{Zu}$ diesem Briefwechsel im Einzelnen siehe KATRIN KeLler, Zwischen zwei Residenzen: Der Briefwechsel der Kurfürstin Anna von Sachsen mit Freiin Brigitta Trautson, in: Helmut Bräuer/Gerhard Jaritz/Käthe Sonnleitner (Hg.), Viatori per urbes castraque. Festschrift Herwig Ebner (Schriftenreihe des Instituts für Geschichte 14), Graz 2003, S. 365382. 
Kaiserin auszurichten, von deren Gesundheit zu berichten und Gaben an die Kaiserin zu übermitteln. ${ }^{32}$ Nach 1570 fehlte dies in keinem Brief, obwohl die Kurfürstin ab August dieses Jahres, wie eben ausgeführt, ja auch eine direkte Korrespondenz mit der Kaiserin unterhielt. Sehr häufig wurden nun ein Brief an Brigitta Trautson und einer an die Kaiserin am gleichen Tag und nicht selten gemeinsam übersandt. Die Freiin Trautson ging dann nach Empfang von Brief und gegebenenfalls damit verbundenen Gaben zur Kaiserin und übergab sie dort in aller ondertanigkhat, wie mier ener curfürstlichen Durchlaucht pefoblen haben. ${ }^{33}$ Sie fungierte also in diesen Jahren kontinuierlich als Mittlerin zwischen Kaiserin (manchmal auch deren Töchtern) und Kurfürstin.

Warum Anna neben der direkten Korrespondenz auch diesen Weg wählte, um Verbindungen zu Kaiserin Maria herzustellen, ist bislang nicht ganz klar. Ein Grund dürfte darin gelegen haben, dass weder die Kaiserin noch ihre Töchter in der Lage waren, eigenhändig deutsche Briefe zu schreiben, ${ }^{34}$ was eine vertraute Korrespondenz verhinderte. Ein möglicher Grund für die Einschaltung einer Vermittlerin könnte auch darin gelegen haben, dass Anna von Sachsen sich über die Art des Umgangs mit der Kaiserin nicht immer ganz sicher war; sie zweifelte offenbar daran, ob ihre Gaben für eine Kaiserin standesgemäß seien, welche Vertrautheit des Umgangs der Kaiserin angenehm sein würde, ${ }^{35}$ und versuchte diese Hürde durch Brigittas Vermittlung zu überwinden. Vielleicht sollte auch die Einschaltung der Freiin Trautson als Gemahlin eines hohen höfischen Amtsträgers Annas Briefen und Gaben zusätzliches Gewicht verleihen. ${ }^{36}$ Sicher ist, dass Brigitta Trautson auf diese Weise das wichtigste Bindeglied zwischen den beiden Fürstinnen darstellte und die Korrespondenz zwischen ihr und Anna von Sachsen somit einen besonderen Stellenwert erlangte.

Ähnlich wie im Briefwechsel mit der Kaiserin selbst finden sich in den 97 vorliegenden Briefen der Korrespondenz zwischen Kurfürstin Anna und Brigitta Trautson zudem Beispiele für Fürbitten, die die Freiin ihrerseits für die Kurfürstin bei ihrem Gemahl oder bei der Kaiserin leistete. Wie in der Korrespondenz mit der Kaiserin fällt auch im Briefwechsel zwischen Kurfürstin Anna und Brigitta Trautson (ebenso wie

32 Z. B. HStA Dresden, 10024 Geheimes Archiv, Copial 512, fol. 202v-203r (2. April 1567); Loc. 8531/2, fol. 217r (20. März 1570); Copial 514, fol. 126v-127r (27. März 1570); Copial 516, fol. 16r/v (6. Februar 1572); Copial 519, fol. 40v-41r (10. Juni 1575).

33 So z. B. HStA Dresden, 10024 Geheimes Archiv Loc. 8531/2, fol. 336r (29. Dezember 1571).

34 Vgl. ebd., fol. 221r (3. April 1570), Brigitta Trautson an Kurfürstin Anna: Gnedigiste Curfürstin ond fraw, ich hab ener curfürstlichen Durchleichtigkhat gnadigist schreiben mit aller vndertanigisten gehorsam vnd referentz empfanngen samt dem schreiben an die Kaiserin, des ich den in der stunt [so]palt ichs empfanngen hab, irer Majestät vberantwurt, vnd die Kaiserin ist gantz wol damit erfreit gebesen mit euer curfürstlichen Durchleichtigkhat schreiben. Ier majestät schreiben auch euer curfürstlichen Durchleichtigkhat wider gern, so khan ier Majestät nit daitz schreiben, euer curfürstlichen Durchleichtigkhat miesen die entschuldiggung zu guet nebmen von ier Majestät.

35 Vgl. HStA Dresden, 10024 Geheimes Archiv, Copial 514, fol. 158v (23. Juli 1570), Kurfürstin Anna an Brigitta Trautson: Vnnd gesinnen gantz gunstig, ir wollet euch solcher mube vmb vnserntwillen nicht beschweren und vns ja wider vermelden, ob wir recht oder vnrecht thun, das wir vns gegen irer Majestet so vertreulich einlassen [...].

36 Die Kurfürstin bat wiederholt um Empfehlung bei Kaiserin und Kaiser, siehe etwa HStA Dresden, 10024 Geheimes Archiv, Copial 512, fol. 122v-123r (12. Juni 1566); Loc. 8534/4, fol. 144r (7. März 1573). 
mit Erzherzogin Maria von Innerösterreich ${ }^{37}$ oder Herzogin Anna von Bayern) auf, dass stets solche Interventionen oder Fürbitten eine Art Kulminationspunkte in den oft jahre- oder jahrzehntelangen Korrespondenzen bildeten. Sie kamen relativ selten vor, nicht weil sie nebensächlich waren, sondern weil sie einen wichtigen Beweggrund für Aufbau und Perpetuierung von persönlichen Kontakten zwischen den Frauen darstellten. Die Instrumentalisierung durfte jedoch innerhalb dieser kommunikativen Konstellationen nicht zu häufig sein; sowohl zwischen Brigitta Trautson und Kurfürstin Anna wie in der Beziehung zu den anderen erwähnten Fürstinnen galt es, das richtige Maß bezüglich möglicher Interventionen zu finden, das dem jeweils erreichten Grad persönlicher Verpflichtung entsprach. ${ }^{38}$

Die Relevanz, welche der Korrespondenz mit Damen im Umfeld der Kaiserin beigemessen wurde, dokumentiert sich auch darin, dass es Nachfolgerinnen für Brigitta Trautson in dieser Mittlerrolle gab: Nach deren Tod übernahm zuerst Sophia de Toledo († 1579) diese Funktion, und zwar auf direkte Bitte der Kaiserin. Als Witwe, wahrscheinlich als Hofmeisterin, gehörte sie in den siebziger Jahren des 16. Jahrhunderts in Wien und nach 1576 in Prag dem Frauenzimmer der Kaiserin-Witwe Maria an. ${ }^{39}$ Noch zu Lebzeiten der Brigitta Trautson begann der Briefwechsel zwischen ihr und der Kurfürstin, der dann mit hoher Dichte bis zum Tod Sophia de Toledos im Herbst 1579 anhielt. Schließlich übernahm die Aufgabe der Vermittlung von Kontakten zwischen der Kurfürstin und der Kaiserin auf einer persönlichen Ebene Maria von Pernstein (um 1538-1595), die Frau beziehungsweise Witwe des böhmischen Kanzlers Wratislaw von Pernstein (1530-1582). Sie war eine geborene Manrique de Lara und als ehemalige Hofdame der Kaiserin mit dieser schon lange vertraut. Briefliche Kontakte zwischen ihr und der sächsischen Kurfürstin bestanden bereits seit 1573 und hielten bis zu Annas Tod im Oktober 1585 an. Vor allem vermittelte Maria in der Zeit nach 1581 die Post zwischen den Fürstinnen, weil sie durch familiäre Beziehungen und Reisen ihres Mannes über regelmäßige Kontakte nach Spanien verfügte. Charakteristisch war auch in diesem weniger intensiven Briefwechsel, dass Maria von Pernstein 1583 ihre langjährigen Kontakte zu Kurfürstin Anna nutzte, um zur Schuldentilgung Teile des Familiensilbers an den finanziell sehr gut ausgestatteten Kurfürsten von Sachsen zu verkaufen. ${ }^{40}$

37 In Dresden überliefert in den Kopialen der Kurfürstin sowie in HStA Dresden, 10024 Geheimes Archiv, Loc. 8538/11 (1581-1585) und Loc. 8538/9 (1575-1585). Zur Person siehe KATRIN KelleR, Erzherzogin Maria von Innerösterreich (1551-1608). Zwischen Habsburg und Wittelsbach, Wien/Köln/Weimar 2012.

38 Vgl. dazu etwa Maurice Godelier, Das Rätsel der Gabe. Geld, Geschenke, heilige Objekte, München 1999, S. 21-23, 64 f., 145 f.; NAtAlie Zemon Davis, Die schenkende Gesellschaft. Zur Kultur der französischen Renaissance, München 2002, S. 58-62; JAN HiRSCHBIEGEL, Étrennes. Untersuchungen zum höfischen Geschenkverkehr im spätmittelalterlichen Frankreich (Pariser historische Studien 60), München 2002, S. 129-131.

39 Vgl. HStA Dresden, 10024 Geheimes Archiv, Copial 519, fol. 156r/v (19. Februar 1576), fol. 252 (14. September 1576). Ihr Ehemann Pedro Alvarez de Toledo († 1552) war Offizier Karls V. und 21 Jahre lang Generalkapitän in Neapel. Zu ihr und ihrer Familie FrIEDRICH W. LEITNER, Die Mager von Fuchsstatt. Aufstieg und Niedergang einer Adelsfamilie in der Frühen Neuzeit, in: Archiv für Diplomatik 40 (1994), S. 205-252, hier S. 221, $230 \mathrm{f}$.

40 Vgl. HStA Dresden, 10024 Geheimes Archiv, Copial 524, fol. 152r/v (7. August 1583); Copial 525, fol. 126v-127r (7. Juni 1583), fol. 138r (28. September 1583) usw.; Loc. 8538/4, fol. 17r/v (30. Mai 1583), fol. 22r (29. Juli 1583), fol. 24 f. (22. September 1583) usw. $\mathrm{Zu}$ Maria von Pernstein vgl. Charlotte Gamber, Die Herren von Pernstein in Böhmen und Mähren, Ms., o. O. 1982, S. 49 f. 
Schließlich bleibt darauf zu verweisen, dass es gleichzeitig mit den genannten Verbindungen weitere Kontakte Annas von Sachsen in die höfische Gesellschaft der kaiserlichen Residenz gab: Die Kurfürstin bedachte nicht nur das Kaiserpaar, sondern auch Ehefrauen hoher höfischer Amtsträger regelmäßig mit ihrem begehrten Aquavitae. ${ }^{41}$ Außerdem korrespondierte sie sporadisch mit solchen Amtsträgern wie dem Geheimen Rat und Reichsvizekanzler Dr. Johann Ulrich Zasius (1521-1570), mit Peter von Mollart († 1576), Hofmeister der Kaiserin, Leonhard IV. von Harrach (15141590), kaiserlicher Geheimer Rat und Obersthofmeister, und später vor allem mit dem ehemaligen Kämmerer des Kaisers Georg Pruskovký z Pruskova (†1584). ${ }^{42}$ Auch mit weiteren Damen der höfischen Gesellschaft wechselte sie Briefe, Rezepte und Geschenke, wie etwa mit Margarita von Dietrichstein (ca. 1535-1609), der spanischen Ehefrau des Obersthofmeisters Kaiser Rudolfs II., ${ }^{43}$ mit Ludmilla von Thurn $(† 1558)$ und Anna von Lobkowitz zu Hassenstein $(† 1587)$ aus Böhmen. Dank der guten Überlieferung des Briefwechsels dieser Fürstin lässt sich also ein ganzes Netzwerk von Verbindungen zwischen dem kaiserlichen und dem kurfürstlichen Hof erkennen.

\section{III.}

Um die Ausdehnung der Korrespondenzbeziehungen der Kurfürstin Magdalena Sibylla zwischen 1656 und 1680, also während der Regierungszeit ihres Gemahls, beschreiben zu können, wurden hier als Stichproben die Neujahrsgrußschreiben der Jahre 1665 und $1677^{44}$ herangezogen. Die kursorische Durchsicht der - weitgehend ungeordneten - Briefkonvolute hatte ergeben, dass derartige Schreiben einen erheblichen Teil der Korrespondenz der Kurfürstin ausmachten, und dass mit geringen Schwankungen alle ihre Korrespondentinnen und Korrespondenten solche Schreiben sandten und also auf diese Weise erfasst werden können. Nicht erörtert werden können hier Intensität und vor allem Dauerhaftigkeit der Korrespondenzen, weil eine Gesamtdurchsicht der 21 Briefkonvolute nicht möglich war.

Auffällig ist an der räumlichen Struktur der Kontakte zunächst, dass die Herkunftsfamilie der Kurfürstin nur in sehr geringem Umfang in ihrer Korrespondenz präsent war. Das hatte natürlich mit genealogischen Zufällen zu tun; mehrere ihrer Geschwister starben früh. Eine Schwester der Kurfürstin, Anna Maria (1609-1680), hatte Johann Anton von Eggenberg, Herzog von Krumau (1610-1649), geheiratet; sie korrespondierte zumindest sporadisch aus Graz und später aus Ödenburg/Sopron mit ihrer Schwester. Auch ihre beiden Söhne Johann Christian (1641-1710) und Johann Seifried (1647-1703) und später deren Ehefrauen standen mit der Kurfürstin in Kontakt. Deutlich zahlreicher war die Familie des Kurfürsten vertreten; Magdalena Sibylla

41 Vgl. HStA Dresden, 10024 Geheimes Archiv, Loc. 8531/2, fol. 217r (20. März 1570); Copial 516, fol. 16r/v (6. Februar 1572); Loc. 8534/4, fol. 210r (18. Januar 1576).

42 Z. B. HStA Dresden, 10024 Geheimes Archiv, Copial 513, fol. 24v-25v (17. März 1568); Loc. 8538/9, fol. 31r (4. April 1573); Copial 519, fol. 154r-156r (18. Februar 1576). Siehe auch Österreichisches Staatsarchiv Wien, Allgemeines Verwaltungsarchiv, Familienarchiv Harrach, Karton 712, unpag. (6. August 1564).

43 Vgl. Friedrich EdelmaYeR, Ehre, Geld, Karriere. Adam von Dietrichstein im Dienst Kaiser Maximilians II., in: Ders./Alfred Kohler (Hg.), Kaiser Maximilian II. Kultur und Politik im 16. Jahrhundert (Wiener Beiträge zur Geschichte der Neuzeit 19), Wien/ München 1992, S. 109-142, hier S. 114 f.

44 Vgl. HStA Dresden, 10024 Geheimes Archiv, Loc. 8569/1 (1666-1669); Loc. 8570/3 (1677-1678). 
wechselte Briefe sowohl mit dessen drei Brüdern wie den drei Schwestern. Über die Schwestern, die nach Holstein-Gottorf, Hessen-Darmstadt und Sachsen-Altenburg geheiratet hatten, entstanden auch zahlreiche Kontakte zu Nichten und Neffen und damit briefliche Beziehungen der Kurfürstin nach Anhalt-Zerbst, Baden-Durlach, Hessen-Homburg oder Württemberg. Insgesamt war etwa die Hälfte der Absenderinnen und Absender von Neujahrsgrüßen mit der Kurfürstin im ersten oder zweiten Grad verwandt, wobei dieser Anteil mit fortschreitendem Lebensalter naheliegender Weise leicht rückläufig gewesen zu sein scheint.

Unter den nicht oder doch nur weitläufig verwandten Korrespondenten waren Angehörige der verschiedenen Linien des Hauses Anhalt sowie der ernestinischen Linien des Hauses Wettin besonders zahlreich vertreten. Dauerhafte Kontakte gab es auch nach Brandenburg und in die Pfalz sowie zu mehreren Linien der Herzöge von Holstein und nach Württemberg. In jedem Jahr befanden sich unter den Absendern der Neujahrsgrüße aber auch Kaiser Leopold I., später auch dessen Gemahlinnen, und die Kaiserin-Witwe Eleonora Gonzaga-Rethel (1631-1686). Regelmäßig meldeten sich außerdem die Kurfürsten von Mainz und Köln sowie häufig der Kurfürst von Bayern, Ferdinand Maria (1636-1679), und dessen Gemahlin Henriette Adelaide (1636-1676).

Damit umspannte das Korrespondenznetz der Kurfürstin von Sachsen in den sechziger und siebziger Jahren des 17. Jahrhunderts das Alte Reich ebenso weitgehend, wie das um 1580 bei Kurfürstin Anna der Fall gewesen war. Man wird angesichts der erwähnten geistlichen Kurfürsten und der regelmäßigen Schreiben von Kaiser Leopold I. selbst sogar sagen dürfen, dass Magdalena Sibylla von Sachsen über ein vollständigeres Netz brieflicher Kontakte innerhalb des Reiches verfügte als ihre Vorgängerin. Nahe$\mathrm{zu}$ alle evangelischen reichsfürstlichen Häuser und die wichtigsten katholischen Fürsten zählten dazu; ganz offensichtlich war deutlicher als im 16. Jahrhundert die Gruppe der regierenden reichsfürstlichen Häuser der Bezugsrahmen des Korrespondenznetzes, obwohl insgesamt in den Briefen auch andere Absender durchaus in Erscheinung traten. ${ }^{45}$ Hinsichtlich des Geschlechts der Absender von Briefen an die Kurfürstin ist kein so deutliches Übergewicht von Frauen feststellbar, wie das im 16. Jahrhundert der Fall gewesen war; vielmehr ist das Zahlenverhältnis weitgehend ausgeglichen. Auffällig ist, dass sehr viele Ehepaare sich, meist in getrennten Schreiben, mit Glückwünschen bei der Kurfürstin in Erinnerung brachten. Dies ließ sich bei Kurfürstin Anna so ebenfalls seltener feststellen. Erhebliche Unterschiede bestanden schließlich auch hinsichtlich der Inhalte und der Intensität der Korrespondenzbeziehungen von Magdalena Sibylla im Vergleich zu Anna. Darauf soll nun in Hinblick auf die Kontakte nach Wien beziehungsweise zu Adligen aus dem habsburgischen Herrschaftsraum etwas genauer eingegangen werden.

\section{$I V$.}

Die Korrespondenz zwischen Kurfürstin Magdalena Sibylla und dem Kaiserhof entsprach in ihren Grundzügen den zentralen Merkmalen ihres Briefwechsels generell: Gewechselt wurden vorrangig Schreiben mit Neujahrswünschen und mit Gratulationen oder Kondolenzen zu wichtigen familiären Ereignissen. So kondolierten etwa 1656 Kaiser Ferdinand III., seine Gemahlin Eleonora und Leopold I. anlässlich des

45 So beispielsweise Gräfin von Dernath, Gemahlin eines kursächsischen Offiziers (HStA Dresden, 10024 Geheimes Archiv, Loc. 8568/1, fol. 46b-46c, 13. Dezember 1667), eine Freiin von Fürstenberg aus Wien (Loc. 8567/4, fol. 127v, 30. November 1657) und die Korrespondenz mit einigen böhmischen Familien (siehe unten Anm. 53 bis 55). 
Todes von Kurfürst Johann Georg I., Magdalenas Schwiegervater. Im folgenden Jahr übermittelte sie ihrerseits Beileidsschreiben anlässlich des Todes von Ferdinand III. an Leopold I., die Kaiserin-Witwe Eleonora Gonzaga-Rethel sowie an Erzherzog Leopold Wilhelm (1614-1662). ${ }^{46}$ In beiden Fällen wurden die entsprechenden Schreiben von Gesandten ${ }^{47} \mathrm{im}$ Zuge von Audienzen überreicht. Außerdem erhielten Gesandte teilweise eigene Kredenzschreiben ${ }^{48}$ an die Kurfürstin beziehungsweise die Kaiserin, die sie bei ihrer jeweiligen Antrittsaudienz zu übergeben hatten. Derartige Mitteilungen umfassen innerhalb der überlieferten Korrespondenz einen ähnlich großen Teil wie die jährlichen Neujahrswünsche. Im Falle des Kaisers kamen noch regelmäßige Kredenzschreiben hinzu, mit denen er der Kurfürstin anlässlich ihrer jährlichen Aufenthalte im Bad im böhmischen Teplitz/Teplice seine Abgesandten empfahl und die Fürstin begrüßte. Stets beauftragte Leopold I. aus diesem Anlass einen adligen Inhaber eines hohen böhmischen Landesamtes mit der Aufwartung bei der Kurfürstin von Sachsen, solange sich diese im Bad aufhielt. ${ }^{49}$

In quantitativer Hinsicht wurde die Korrespondenz der Kurfürstin im 17. Jahrhundert also von Komplimentenschreiben ${ }^{50}$ dominiert, die in ihren Anlässen und Formulierungen zeremoniellen Regeln folgten. Neben diesen formalisierten Schreiben finden sich aber auch im Briefnachlass von Magdalena Sibylla, allerdings in sichtlich geringerer Frequenz als bei Kurfürstin Anna im 16. Jahrhundert, Schreiben, die mit einem bestimmten Anliegen an sie gerichtet wurden beziehungsweise die auf ein solches Schreiben von ihr reagierten. Dabei wird dann erkennbar, dass sich wiederum mindestens zwei Ebenen der Korrespondenz mit Wien beziehungsweise mit Personen in den habsburgischen Ländern beschreiben lassen. Zum einen gab es neben den erwähnten Courtoisieschreiben einen zumindest sporadischen Briefwechsel mit dem Kaiser selbst; von den Kaiserinnen erscheinen in unseren Stichproben nur ganz selten zusätzliche Schreiben.

Der Briefwechsel mit Leopold I. war auch mit Fürbitten beziehungsweise Bitten um Interzessionen verbunden und teilweise von Gaben begleitet, ähnlich wie wir es bei Kurfürstin Anna feststellen konnten. So empfahl etwa Leopold I. im Dezember 1660

46 Vgl. HStA Dresden, 10024 Geheimes Archiv, Loc. 8567/5, fol. 70r-72r (30. Oktober bzw. 1. November 1656); Loc. 8567/6, fol. 14r-17r, 25r-26r, 71r (April 1657, 3. Juni 1657).

47 Vgl. etwa den ausführlichen Bericht des kursächsischen Gesandten Hans Friedrich von Burckersrode über seine Audienz bei der Kaiserin-Witwe 1660 in HStA Dresden, 10024 Geheimes Archiv, Loc. 8567/4, fol. 35er-36v (16./26. Mai 1660). Teilweise, etwa bei den Neujahrsgrüßen, erfolgte die Übermittlung auch durch den sächsischen Residenten in Wien, Jonas Schrimpf († 1695), z. B. Loc. 8241/1, fol. 74r, 157r, 267v.

48 Vgl. etwa HStA Dresden, 10024 Geheimes Archiv, Loc. 8568/1, fol. 50cr (1. Februar 1667); Loc. 8569/1, fol. 31dr, 32r (18. Februar 1665).

49 Vgl. z. B. HStA Dresden, 10024 Geheimes Archiv, Loc. 8567/6, fol. 52r (3. Mai 1657); Loc. 8568/1, fol. 21r, 43r (23. April 1664), fol. 75 (27. Mai 1673); Loc. 8569/1, fol. 23r (29. April 1665); Loc. 8570/3, fol. 17 (12. Mai 1677). Diese Badereisen sächsischer Fürstinnen können seit dem 16. Jahrhundert nachgewiesen werden: HStA Dresden, 10024 Geheimes Archiv, Loc. 8545/4, unpag. (5. Mai 1593). Zu den Aufenthalten in Teplitz siehe auch: KATRin Keller/Alessandro Catalano (Hg.), Die Diarien und Tagzettel des Kardinals Ernst Adalbert von Harrach. Edition und Kommentar (Veröffentlichungen der Kommission für neuere Geschichte Österreichs 104/1-7), Wien/Köln/Weimar 2010, Bd. 6, S. 315, 622 f., 634; Bd. 7, S. 53, 210, 341, 480, 488, 490, 502, 617, 625, 684.

50 Vgl. ReinHARD NiCKISCH, Die Stilprinzipien in den deutschen Briefstellern des 17. und 18. Jahrhunderts. Mit einer Bibliographie zur Briefschreiblehre (1474-1800) (Palaestra. Untersuchungen aus der deutschen und englischen Philologie und Literaturgeschichte 254), Göttingen 1969, S. 68. 
auf deren Bitte die Witwe eines Offiziers, die wegen eines Rechtsstreits nach Dresden reiste, der Fürsprache der Kurfürstin. Drei Monate später sendete er ein Bologneser Hündchen nach Dresden, weil er erfahren hatte, dass Magdalena Sibylla ein solches wünschte. ${ }^{51}$ Und 1678 bat Kaiserin Eleonora Magdalena (1655-1720) die Kurfürstin um Unterstützung für die Angelegenheiten einer Gräfin Kinský (Elisabeth Maria, geb. Kilagrew), die in Dresden wegen ihres verstorbenen Gemahls noch finanzielle Ansprüche geltend zu machen hatte. Magdalena Sibylla ihrerseits bat 1666 den Kaiser, ihren italienischen Kammermusikus an den Vizekönig von Neapel zu empfehlen, weil dort familiäre Angelegenheiten des Musikers zu klären waren.52 Zugunsten von Ludmila Kinský, Witwe eines prominenten böhmischen Exulanten am kursächsischen Hof, intervenierte die Kurfürstin sowohl bei der Landtafel in Prag wie beim kaiserlichen Obersthofmeister Fürst Johann Ferdinand von Portia (1605-1665) und beim böhmischen Oberstkanzler Johann Hartwig von Nostitz (1610-1683) in Wien.53 Andererseits erbat Gräfin Margaretha von Clary, bei der die Kurfürstin in Teplitz mehrfach Quartier genommen hatte, Magdalena Sibyllas Beistand in einer Schuldsache ihres Vetters Graf Johann Georg von Clary und Aldringen. ${ }^{54}$

Damit ist schon die zweite Ebene der Korrespondenz angesprochen: Verschiedene Damen und Herren vornehmlich des böhmischen Adels standen mit der Kurfürstin in Verbindung. Bei diesen Vertretern und Vertreterinnen beispielsweise der Familien Clary und Aldringen, Martinitz, Kolovrat sowie bei Matthäus Ferdinand Sobek von Bilenburg (1618-1675), Erzbischof von Prag, ${ }^{55}$ handelte es sich meist um Personen, die die Kurfürstin bei ihren Reisen nach Teplitz kennengelernt hatte. Kontakte knüpfte sie aber beispielsweise auch, als sie 1658 ihren Gemahl zur Kaiserwahl nach Frankfurt am Main begleitete. ${ }^{56}$ Neben Ersuchen um Fürsprache wie im oben erwähnten Fall der Gräfin von Clary waren diese Briefwechsel auch mit der Übernahme von Patenschaften durch die Kurfürstin und kleineren Geschenken verbunden. ${ }^{57}$

Eine spezielle Ebene dieser Kontakte nach Wien beziehungsweise in die Erblande stellte natürlich die Korrespondenz mit Magdalena Sibyllas Schwester Anna Maria von Eggenberg dar. Sie unterschied sich von den bisher erwähnten Verbindungen nicht unbedingt durch ihre Frequenz; von einem vertrauten Briefwechsel kann nicht gesprochen werden. Trotzdem sind diese Briefe in ihrem Charakter eindeutig Familienbriefe; es geht um die problematische Position der Fürstin in Graz, die sich aus ihrem lutherischen Bekenntnis ergab und die schließlich dazu führte, dass Anna Maria von Eggenberg 1671 ins ungarische Ödenburg/Sopron übersiedelte, wo sie weitgehendere Frei-

51 Vgl. HStA Dresden, 10024 Geheimes Archiv, Loc. 8568/1, fol. 4r (15. Dezember 1660), fol. 5r (13. März 1661).

52 Vgl. HStA Dresden, 10024 Geheimes Archiv, Loc. 8570/3, fol. 107r (26. März 1678); Loc. 8569/1, fol. 156r (21. März 1666).

53 Vgl. HStA Dresden, 10024 Geheimes Archiv, Loc. 8570/1, fol. 6r (10. Juli 1671), fol. 36r (30. Juli 1664); Loc. 8570/3, fol. 107r (26. März 1678).

54 Vgl. HStA Dresden, 10024 Geheimes Archiv, Loc. 8570/3, fol. 112r (22. Mai 1678).

55 Vgl. HStA Dresden, 10024 Geheimes Archiv, Loc. 8568/1, fol. 21r (23. April 1664), fol. 75r (27. Mai 1673); Loc. 8569/1, fol. 13r (5. Januar 1665), fol. 48cr, 49cr (13. September 1670); Loc. 8570/1, fol. 6r (10. Juli 1671), fol. 27r (23. April 1673).

56 Vgl. HStA Dresden, 10024 Geheimes Archiv, Loc. 8567/4, fol. 150br, 150er (11. September 1658).

57 Vgl. HStA Dresden, 10024 Geheimes Archiv, Loc. 8568/1, fol. 126r (13. August 1686); Loc. 8570/1, fol. 122r (30. April 1674), fol. 133r (23. August 1674). 
heiten nutzen konnte. ${ }^{58}$ Es geht aber auch um Erbschaftsfragen, die Verehelichung der beiden Söhne der Fürstin etc. Mit diesen beiden Söhnen dagegen tauschte Kurfürstin Magdalena Sibylla fast ausschließlich Komplimentenschreiben aus.

Hinzuweisen bleibt schließlich noch auf eine Ebene von Kontakten Magdalena Sibyllas, die sich in ihrer Korrespondenz kaum widerspiegeln, die aber trotzdem Verbindungen der Kurfürstin in die Habsburgermonarchie begründeten. Da nicht im Einzelnen nachzuvollziehen ist, wen sie bei ihren Badaufenthalten in Böhmen traf, ist die Mitteilung, dass Magdalena Sibylla gemeinsam mit ihrer Tochter im Sommer 1658 der Hochzeit des (katholischen) Markgrafen Leopold Wilhelm von Baden-Baden (1626-1671) mit Sylvia Katharina Czernin (1606-1664) in Lobositz/Lovosice beiwohnte ${ }^{59}$ einer der wenigen Hinweise darauf, dass sich aus Bekanntschaften durchaus engere Kontakte entwickeln konnten, die das Verbindungsnetzwerk der Fürstin bereicherten. Dies gilt auch für ihre Beziehungen zu Hofdamen, wobei sich gerade in der Zeit Magdalena Sibyllas in Dresden wiederholt Mädchen aus böhmischen und österreichischen Exulantenfamilien in ihrem Hofstaat befanden. ${ }^{60}$ Über sie und ihre Verwandten waren weitere Kontakte nach Wien möglich.

V.

Insgesamt lässt sich also festhalten, dass die beiden Kurfürstinnen in ihrer Korrespondenz einander insoweit glichen, als sie sowohl direkt mit dem Kaiserhaus wie mit adligen Familien der Erblande in brieflicher Verbindung standen. Im 17. Jahrhundert scheint die Frequenz der Briefwechsel insbesondere mit der kaiserlichen Familie in quantitativer Hinsicht sogar relativ höher gewesen zu sein als zur Zeit von Kurfürstin Anna. Für beide Kurfürstinnen kann festgehalten werden, dass ihre Korrespondenzen ganz offensichtlich Bestandteil eines engmaschigen Netzes von Kommunikation im Alten Reich waren, das sich im 16. und 17. Jahrhundert jedoch durchaus unterschiedlich darstellte. Für Kurfürstin Anna unterstreicht eine Parallelisierung der Korrespondenzbeziehungen der Kurfürstin mit politischen Gruppierungen des fraglichen Zeitraums, dass Fürst und Fürstin einander als Arbeitspaar bei der Aufrechterhaltung ,guter Korrespondenz' mit Nachbarn und Verbündeten ergänzten. ${ }^{61}$

Die hier vorgestellten Korrespondenzbeziehungen des 16. Jahrhunderts lassen sich außerdem in einen speziellen Aspekt der Vernetzung zwischen Höfen und Residenzen des Alten Reiches einordnen, in das Phänomen der Klientelbildung über die eigene höfische Gesellschaft hinaus. ${ }^{62}$ Betrachtet man den Briefwechsel zwischen Anna von

58 Vgl. HStA Dresden, 10024 Geheimes Archiv, Loc. 8567/4, fol. 29r (14. Mai 1649), fol. 88r (9. August 1651); Loc. 8568/ 1, fol. 68cr (22. Oktober 1670), fol. 70r (1. Juli 1671); Loc. 8569/1, fol. 25r (8. Mai 1665), fol. 36r (29. Mai 1665), fol. 54r-55r (September 1665).

59 Keller/Catalano, Diarien und Tagzettel (wie Anm. 49), Bd. 6, S. 638 f., 642 f.

60 Susanna Elisabeth von Oppersdorf, Kammerfräulein, verehelichte Schleinitz (HStA Dresden, 10006 Oberhofmarschallamt, B 22, 29. Februar 1660); Judith Dorothea Jörger, Kammerfräulein, verehelichte Körbitz und Johanna Susanna von Neydegg, Kammerfräulein, verehelichte Degenfeld (HStA Dresden, 10006 Oberhofmarschallamt, B 23, 1674).

61 LutTenberger, Kurfürsten (wie Anm. 2), S. 446; HeIde Wunder, Herrschaft und öffentliches Handeln von Frauen in der Gesellschaft der Frühen Neuzeit, in: Ute Gerhard (Hg.), Frauen in der Geschichte des Rechts. Von der Frühen Neuzeit bis zur Gegenwart, München 1997, S. 27-54, hier S. 32, 35 f.; Keller, Kommunikationsraum (wie Anm. 11), S. 227.

62 Zur Relevanz von Netzwerken für die Handlungsfähigkeit fürstlicher Frauen siehe KATRIN Keller, Mit den Mitteln einer Frau - Handlungsspielräume adliger Frauen in Poli- 
Sachsen und Kaiserin Maria sowie anderen Personen des kaiserlichen Hofes, so können vor allem die zwischen der Kurfürstin und der Kaiserin vermittelnden Frauen mit weitgehender Sicherheit einer Klientel der sächsischen Kurfürstin in Wien zugeordnet werden. Deutlicher Ausdruck dieses Verhältnisses sind - neben dem Briefwechsel an sich - der damit verbundene Gabentausch sowie die Bitte um und Gewährung von Fürbitten. ${ }^{63}$ Zugleich waren Brigitta Trautson, Sophia de Toledo und Maria von Pernstein über familiäre Verbindungen und ihre Funktionen als Ehefrauen bedeutender Räte und Hoffunktionäre auch in Netzwerke des Wiener Hofes eingebunden. Wie dies konkret aussah, entzieht sich freilich bisher unserer Kenntnis.

Für Kurfürstin Magdalena Sibylla sind zwar ebenfalls Gaben und Fürbitten auszumachen, die aus unseren Stichproben erkennbare Frequenz der damit verbundenen Korrespondenzen macht es jedoch unwahrscheinlich, dass sich auch für sie regelrechte Klientelbeziehungen nachweisen lassen werden. Zudem waren ihre Kontakte nicht gezielt auf das Umfeld der Kaiserin oder in die höfische Gesellschaft Wiens gerichtet, sondern beschränkten sich - wohl nicht zuletzt aufgrund der regelmäßigen Badereisen der Kurfürstin - eher auf Böhmen. In beiden Fällen ist allerdings erkennbar, dass persönliche Bekanntschaft für die Inanspruchnahme der Fürsprache der Fürstin beziehungsweise für deren Möglichkeiten der Einflussnahme entscheidend war. Angesichts rückläufiger direkter Kontakte durch Reisen war Magdalena Sibylla auch deshalb in einer schwierigeren Position als Kurfürstin Anna. Für Letztere boten ihre Beziehungen zu Damen des Wiener Hofes sowie zur kaiserlichen Familie die Möglichkeit, über ihre direkte Umgebung in Dresden hinaus und relativ unabhängig von entsprechenden Netzwerken ihres Mannes eigene Verbindungen zum kaiserlichen Hof aufzubauen. Diese konnte sie für ihre sächsische Klientel einsetzen und manchmal sogar direkt politisch nutzbar machen. ${ }^{64}$ Für Magdalena Sibylla dagegen gibt es bislang keinerlei Hinweis auf eine Nutzung der Korrespondenzbeziehungen in dieser Hinsicht. Über die ,klassischen' Handlungsfelder der Fürstin wie Ehestiftung, Fürbitten, Patenschaften scheint sie nicht hinausgegangen $\mathrm{zu}$ sein.

Schließlich bleibt noch ein Bereich zu erwähnen, der in Annas Korrespondenzen mit Personen am Kaiserhof eine Rolle spielte, bei Magdalena Sibylla aber nicht greifbar wird, der Transfer höfischer Kulturgüter und Luxuswaren. Die mehrfach erwähnten Geschenke, mit denen beide Seiten die Korrespondenzbeziehungen festigten und unterstützten, sind in diesem Zusammenhang von teilweise hohem kulturgeschichtlichen Interesse: Die Kurfürstin nutzte etwa ihre Kontakte zu Brigitta Trautson, um bereits 1567 Tulpenzwiebeln für den Dresdner Hofgarten zu beschaffen; mehrfach werden Samen spanischer und amerikanischer Pflanzen oder veredelte Bäume beziehungsweise Pfropfreiser erwähnt, die die Kurfürstin als Geschenk erhielt oder

tik und Diplomatie, in: Hillard von Thiessen/Christian Windler (Hg.), Akteure der Außenbeziehungen. Netzwerke und Interkulturalität im historischen Wandel, Köln/Weimar/ Wien 2010, S. 219-244, hier S. 235-240.

63 BARBARA HARRIS, Women and Politics in Early Tudor England, in: Historical Journal 33 (1990), S. 259-281, hier S. 265-269; AmAlie Fössel, Die Königin im mittelalterlichen Reich. Herrschaftsausübung, Herrschaftsrechte, Handlungsspielräume, Darmstadt 2000, S. $123-150$.

64 Vgl. HStA Dresden, 10024 Geheimes Archiv, Copial 519, fol. 155r-156r (18. Februar 1576); eine gleiche Bitte an Georg Proskowski von Proskau: Copial 519, fol. 154r-155r (18. Februar 1576). 
bestellte. ${ }^{65}$ Auch der Tabak kam 1585 über Wien nach Dresden. ${ }^{66}$ Anna bezog freilich auch Informationen über den Ablauf höfischer Festlichkeiten aus den Briefen der Wiener Damen, ließ sich 1575 im Vorfeld des kaiserlichen Besuches in Dresden über zeremonielle Aspekte des Empfangs informieren und kaufte Handschuhe in Innsbruck. ${ }^{67}$ Es konnten also neben politischen Erwägungen auch kulturelle Funktionen des Kaiserhofs im Reich und für das Reich sein, die in der Korrespondenz von Fürstinnen ihren Niederschlag fanden.

Im Vergleich der Briefwechsel der beiden Fürstinnen mit dem Kaiserhof sowie in ihrer räumlichen Struktur treten somit Gemeinsamkeiten wie Unterschiede zutage. Bei deren Einordnung sind freilich auch zeittypische Ausprägungen der Briefkultur zu berücksichtigen, folgten doch die Briefe des 17. Jahrhunderts anderen Regeln der „Zierlichkeit“. ${ }^{68}$ Die Normierung der Briefkultur, die zeremoniell-ständische Prägung der Korrespondenz von Kurfürstin Magdalena Sibylla ist deutlicher erkennbar als bei Kurfürstin Anna und spielte sowohl bei Anlässen für Briefe wie bei deren Ausgestaltung und vermutlich auch bei der Auswahl der Adressaten und Adressatinnen eine größere Rolle. Zumindest fällt die Beschränkung der Korrespondenz von Kurfürstin Magdalena Sibylla auf einen (hoch-)adligen Kreis im Vergleich zu der von Kurfürstin Anna deutlich ins Auge. Ein umfassenderer Vergleich beider Briefnachlässe müsste diesen Eindruck freilich noch differenzieren.

Der hier versuchte Vergleich hat meines Erachtens vor allem auch deutliche Unterschiede zwischen beiden Fürstinnen hinsichtlich ihrer Aktivitäten, ihres Gestaltungswillens in Bezug auf die Korrespondenzbeziehungen zutage gefördert. Das Fehlen der ausgehenden Briefe für Kurfürstin Magdalena Sibylla macht die Einschätzung zwar schwierig, aber im Gegensatz zu Kurfürstin Anna lassen sich weder Anzeichen für die bewusste Herstellung von brieflichen Kontakten noch für deren Instrumentalisierung im Sinne politischer Einflussnahme erkennen. Es wäre zu prüfen, ob und inwieweit dies auch mit der im Vergleich zu Kurfürst August reichspolitisch viel diffizileren Stellung Kurfürst Johann Georgs II. zusammenhing. In erster Linie dürfte sich hier aber ein individueller Unterschied der beiden Frauen abbilden: Anna nutzte die ihr gegebenen Handlungsspielräume bewusst aus, während Magdalena Sibylla wahrscheinlich beispielhaft für die nicht geringe Zahl von Fürstinnen stehen kann, denen eine aktive Rolle im politischen Geflecht des Alten Reiches nicht erstrebenswert schien oder die an der Aneignung derartiger Handlungsfelder gehindert wurden. Annas ausgedehnte Korrespondenz dagegen ist als Zeichen und als Niederschlag ausgedehnter Aktivitäten im Kontext der Herrschaftsausübung des Fürstenpaares zu betrachten. Dass es sich bei

65 Vgl. HStA Dresden, 10024 Geheimes Archiv, Loc. 8531/2, fol. 140r (7. April 1567); Copial 512, fol. 203r (2. April 1567); Loc. 8534/4, fol. 90r/v (5. September 1573); Loc. 8530/3, fol. 49r (23. Juni 1571); Copial 516, fol. 39v-40r (9. April 1572); Loc. 8435/1, fol. 223r-224r (17. September 1581). Vgl. auch Christopher F. LAFERL, Die Kultur der Spanier in Österreich unter Ferdinand I. 1522-1564, Wien/Köln 1997, S. 148-150. Zu Annas agrarwirtschaftlichen Interessen siehe zuletzt URSULA SCHLUDE, Agrarexpertin am fürstlichen Hof. Überlegungen zur Sozial- und Geschlechtergeschichte des Agrarwissens in der Frühen Neuzeit, in: Zeitschrift für Agrargeschichte und Agrarsoziologie 56 (2008), S. 33-48.

66 Vgl. HStA Dresden, 10024 Geheimes Archiv, Copial 527, fol. 114r/v (3. Juni 1585), fol. 115v-116r (6. Juni 1585); Loc. 8538/4, fol. 79r/v (6./16. Juni 1585), fol. 82r/v (7./17. Juni 1585).

$67 \mathrm{Vgl}$. Keller, Zwischen zwei Residenzen (wie Anm. 31), S. 372 f.

68 Nickisch, Stilprinzipien (wie Anm. 50), S. 30, 72 f., 76. 
ihr um eine ungewöhnlich aktive und einflussreiche Fürstin handelte, wurde bereits von einigen ihrer Zeitgenossen - nicht immer positiv ${ }^{69}$ - angesprochen.

Ungeachtet dieser Unterschiede bleibt freilich festzuhalten, dass in beiden Fällen die untersuchten Briefwechsel zur Verdichtung der Kommunikation zwischen den Höfen des Alten Reiches beitrugen. Der Kaiserhof spielte dabei auch für protestantische Reichsfürsten eine besondere Rolle, wie die Struktur der Korrespondenzen beider Fürstinnen sichtbar werden ließ. Das Alte Reich als Kommunikationsraum erlebte zwar im 16. Jahrhundert möglicherweise eine besondere Blüte, auch im 17. Jahrhundert wäre jedoch die Vernetzung der Höfe mit Wien und über Wien sowie untereinander weiter zu untersuchen. Den Korrespondenzen von Fürstinnen kam dabei ein fester Platz zu.

69 Vgl. Keller, Familie und Politik (wie Anm. 16), S. 291 f.; Keller, Kurfürstin Anna (wie Anm. 13), S. 110 f., 135-138. 